\title{
PULSED DOPPLER-FREE TWO-PHOTON SPECTROSCOPY OF POLYATOMIC MOLECULES
}

\author{
E. RIEDLE, R. MODER and H.J. NEUSSER \\ Institut für Physikalische and Theoretische Chemie, Technische Universität München, \\ D-8046 Garching, Fed. Rep. Germany
}

Received 24 June 1982

\begin{abstract}
Doppler-free two-photon electronic spectra of a large polyatomic molecule are recorded for the first time with pulsed laser radiation of near Fourier-transform limited bandwidth ( $\Delta \nu \sim 100 \mathrm{MHz})$. The resolution obtained is sufficient to resolve individual rotational lines. Due to the high density of these rotational transitions a strong Doppler-broadened background is observed, which is, however, substantially reduced by suitable choice of photon polarizations. Different vibronic bands of benzene $\left(\mathrm{C}_{6} \mathrm{H}_{6}\right)$ are investigated and very accurate rotational constants are found.
\end{abstract}

\section{Introduction}

Doppler-free two-photon absorption was predicted to yield extremely high resolution spectra as early as 1971 [1]. Until recently this had been realized experimentally only for atoms $[2,3]$ and small molecules [4].

In our recent work, however, we have demonstrated that it is also possible to obtain Doppler-free electronic two-photon spectra of large polyatomic molecules over a broad spectral range containing several hundreds of rotational lines [5]. These spectra have been taken by focusing two counter-propagating $\mathrm{cw}$ laser beams into the molecular gas. One photon for the two-photon absorption process originated from an $\mathrm{Ar}^{+}$ion laser with fixed frequency and high in tensity whereas the second photon was from a tunable $\mathrm{cw}$ dye laser of lower intensity. It is the intensity of the fixed frequency laser which makes the weak twophoton absorption observable even with the modest intensity of a cw laser system, at present the only system able to provide the desired frequency resolution in the $\mathrm{MHz}$ range. For weakly fluorescing molecules, however, it would be desirable to have an extremely narrowband pulsed tunable laser with a peak intensity higher by some order of magnitude and thereby increase the probability of the nonlinear twophoton process. Pulsed dye oscillator systems normal- ly are limited in resolution to about $1 \mathrm{GHz}$ and, more severely, if an additional external filter interferometer is added for further line narrowing [6] smooth continuous scans, which are absolutely necessary for obtaining a molecular spectrum, are difficult to obtain.

In this work we present the first Doppler-frec twophoton electronic spectrum of a large molecule $\left(\mathrm{C}_{6} \mathrm{H}_{6}\right)$ obtained with a pulsed highly intense laser source. Since two equal photons are used for the two-photon absorption process the problem of a Dopplerbroadened background from the absorption of two photons from one laser beam arises. This is a severe problem in Doppler-free spectroscopy of large molecules since here many lines are located within the Doppler-width and the intensity ratio of Doppler-free and Doppler-limited background a priori is not as good as in atomic spectra where only few lines are located within the Doppler-width. It will be shown how photon polarization can be chosen in order to suppress this Doppler-broadened background.

\section{Experimental set-up}

The scheme of the experimental set-up is shown in fig. 1. The high resolution and the possibility for a precise linear scan is provided by a cw ring laser system (Coherent 699). The ring laser operates around 


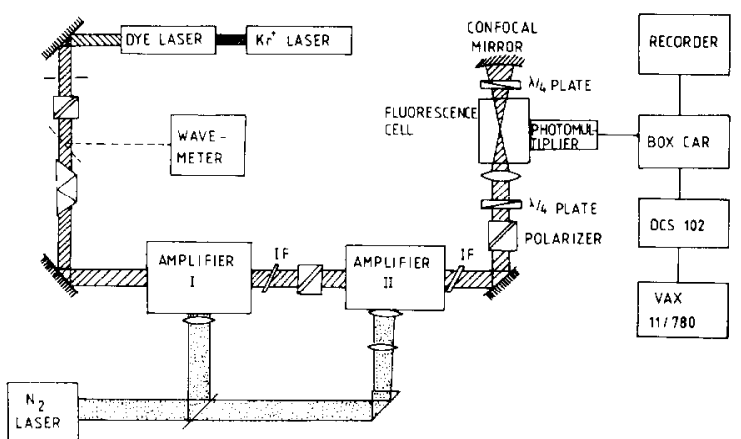

Fig. 1. Experimental set-up for recording Doppler-free twophoton electronic spectra of $\mathrm{C}_{6} \mathrm{H}_{6}$. The single-mode cw-dye laser output is amplified in two stages pumped by the pulsed light of the $\mathrm{N}_{2}$ laser. The resulting light pulses are used for the excitation of the molecules.

$4900 \AA$ with a Coumarin 102 dye and is pumped by the violet lines of $\mathrm{Kr}^{+}$ion laser (CR $3000 \mathrm{~K} \mathrm{UV}$ ). At this wavelength the output power is about 100 $\mathrm{mW}$. The wavelength of the $\mathrm{cw}$ light is continuously controlled by a wavemeter (Burleigh WA-20). In this way absolute wavelengths with an accuracy of 0.03 $\mathrm{cm}^{-1}$ or $1 \mathrm{GHz}$ are obtained.

The cw laser system is optically decoupled from the amplifier system by a direct vision prism. For amplification we use a dye amplifier system pumped by a nitrogen laser (Molectron UV 1000) with a pump power of about $700 \mathrm{~kW}$. The general design of our amplifier system corresponds to that recently published by Salour [7]. We are, however, using narrow band transmission interference filters (FWHM: $30 \AA$ ) for decoupling the two amplifier stages and suppressing stimulated emission from the amplifier stages. The transmission maximum can be easily tuned over a wavelength range of $300 \AA$ by tilting the filter. For simplicity only two amplifier stages ( $\mathrm{C} 485$ in dioxane) have been used yielding $30 \mathrm{~kW}$ light pulses with a pulse length of $5 \mathrm{~ns}$ and a nearly Fourier transform limited bandwidth of $100 \mathrm{MHz}$. The total power of the broad stimulated emission is by a factor of 20 smaller than the power of the narrow band amplified light. This ratio is by far sufficient to guarantee that there is no background due to a two-photon absorption of the broad-band stimulated emission.

The pulsed narrow bandwidth laser light is focused into a fluorescence cell with a low stray light level containing the molecular gas $\left(\mathrm{C}_{6} \mathrm{H}_{6}\right)$ under a pressure of 4 torr. The divergent light beam is then refocused by an adjustable confocal mirror so that a complete overlap of the opposite light beams is obtained. By inserting a Glan polarizer and in addition a quarter wave plate the light beam is either linearly or circularly polarized. The sense of the circular polarization of the backward reflected light beam can be changed by inserting a second $\lambda / 4$ retardation plate.

The UV fluorescence from the two-photon excited molecules is observed with a solar blind $(9 \mathrm{~mm}$ UG 5 Schott filters) 56 DUVP photomultiplier whose signal is fed into a box car integrator. The intcgrated signal is recorded on a strip chart recorder and at the same time transferred to a DCS 102 data processing system. There it is digitized, stored on floppy discs and then transferred to a VAX 11/780 computer. This procedure enables us to obtain the complete twophoton spectrum (made up from several individual overlapping scans of the dyc laser) with suitable scaling factors.

\section{Experimental results and discussion}

\subsection{Polarization behaviour and Doppler-broadened background}

In fig. 2 and fig. 3 the high-resolution two-photon spectrum of $\mathrm{C}_{6} \mathrm{H}_{6}$ vapour is shown as measured with the pulsed light source and diverse polarization conditions. In both figures a small part of the $S_{1} \leftarrow S_{0}$ spectrum is shown. This is the blue edge of the Qbranch $(\Delta J=0)$ of the totally-symmetric $\left(\mathrm{A}_{1 \mathrm{~g}}\right)$ ${ }_{14}^{1} 1_{0}^{1}$ [8] transition induced by the vibration $\nu_{14}$ $\left(b_{2 u}\right)$ in the ${ }^{1} B_{2 u}$ clectronic state.

To record fig. $2 \mathrm{~b}$ only one (circularly polarized) laser beam is used. The two photons absorbed have the same direction of propagation and therefore a Doppler-broadened (Doppler-width 1.7 GHz) spectrum is seen.

For fig. 2a the light wave is reflected back into the fluorcsccnce cell. While the incoming wave has right hand circular polarization, the reflected wave is then left hand circularly polarized. However, in the molecular reference frame the two beams are polarized in the same sense ( $\mathrm{Q} 2)$.

A comparison of fig. $2 a$ and $2 b$ immediately reveals, that the broad structure in fig. $2 a$ is Doppler- 


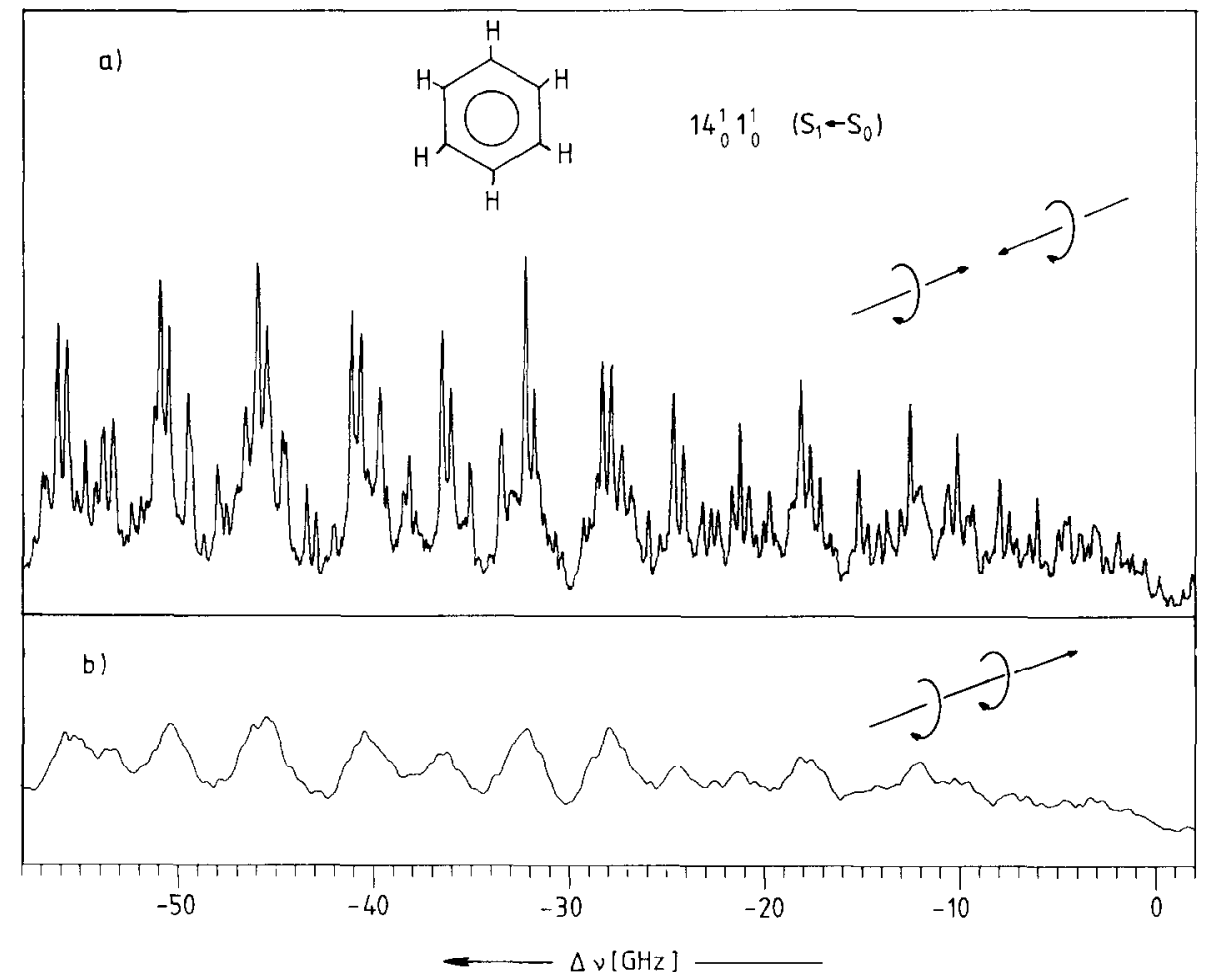

Fig. 2. Part of the two-photon electronic $S_{1} \leftarrow S_{0}$ spectrum of $C_{6} H_{6}$. The blue edge of the Q-branch of the $14_{0}^{1} 1_{0}^{1}$ band is shown. If recorded a) with one (circularly polarized) laser beam a Doppler-broadened spectrum is seen, b) with two oppositely propagating beams also Doppler-free contributions are seen.

broadened background due to the absorption of two photons from one laser beam. The sharp peaks on top of this broad background represent the Doppler-free spectrum of the molecule obtained by the absorption of one photon each from the two oppositely propagating light beams.

A signal stronger by one order of magnitude is obtained when both photons are linearly polarized. The spectrum recorded in this way $(\$ \uparrow)$ is shown in fig. 3a whereas in fig. $3 \mathrm{~b}$ both photons (in the molecular system) are circularly polarized in the opposite sense (DS). The spectra in fig. $3 a$ and $b$ and identical, the only difference being an additional strong Dopplerbroadened background in the case of the linear $(\$ \uparrow)$ polarization.

Even though in both figs. 2 and 3 the same frequency range of the $\mathrm{C}_{6} \mathrm{H}_{6}$ spectrum is shown the measured Dopper-free and Doppler-broadened spectra are completely different under different polarization conditions. A Doppler-free spectrum not perturbed by the
Doppler-broadened background is only observed for the case of counter circularly polarized light (২૬) shown in fig. 3a.

For an explanation of these results we consider the general expression for the two-photon absorption probability $[9,10]$. As in the Raman effect there exist three tensor contributions to the two-photon transition probability: the isotropic contribution, the antisymmetry anisotropic contribution, and the symmetric anisotropic contribution. The different contributions have been investigated previously theoretically and experimentally for liquids [11] and for gases $[9,10]$. For this work it is important that the isotropic contribution is only existent for the Q-branch $(\Delta J=0)$ of totally symmetric two-photon transitions and is only observed for the photon polarizations $\$ \uparrow, 2 S$, and $\downarrow \geq$. On the other hand symmetric anisotropic contributions are existent for all allowed rotational branches $\Delta J= \pm 2, \pm 1,0$ of all two-photon transition symmetries while antisymmetric contributions are 


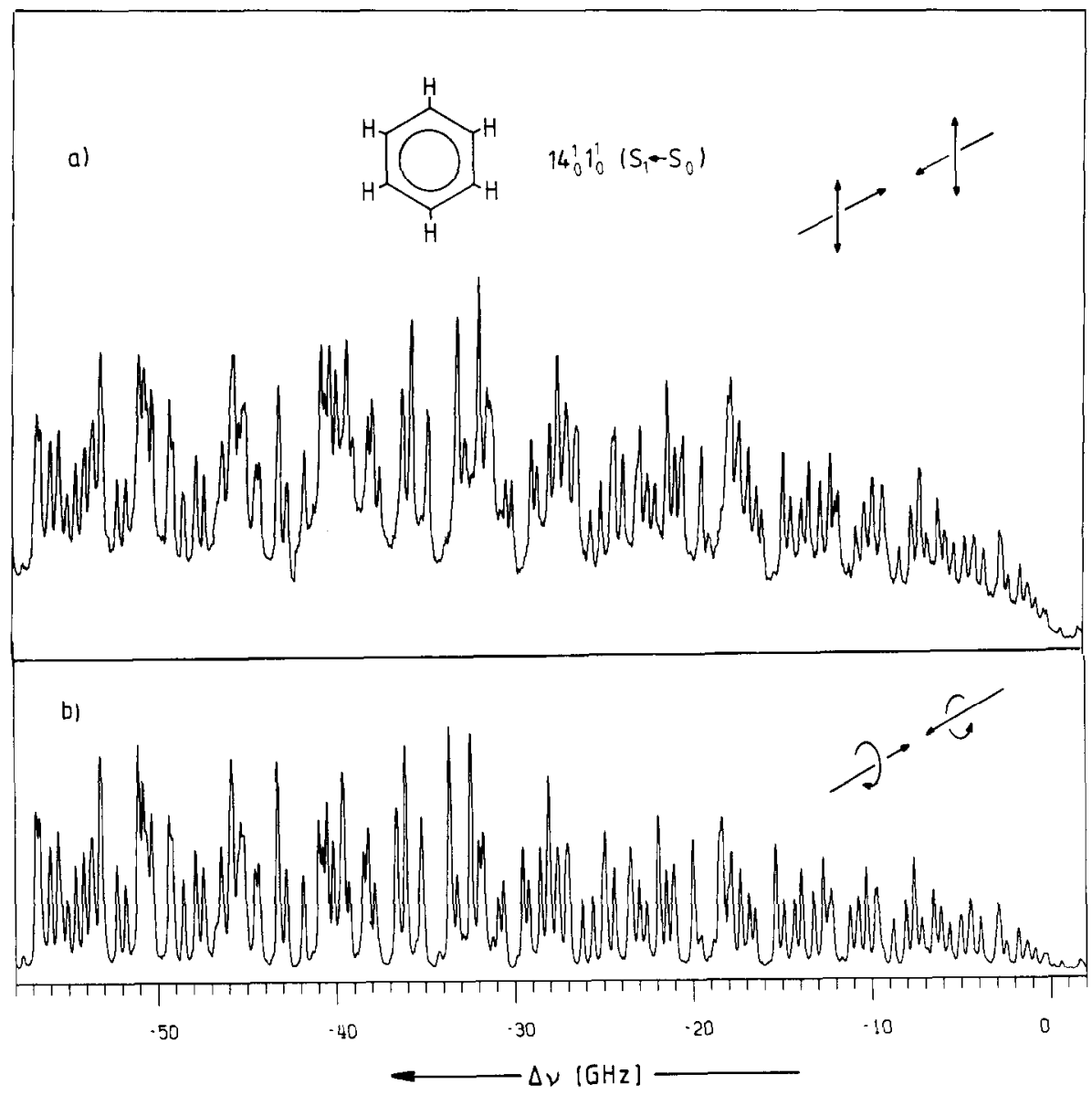

Fig. 3. Same frequency range of the $\mathrm{C}_{6} \mathrm{H}_{6}$ two-photon spectrum as in fig. 2. However, different polarizations of the two laser beams were used. In a) linear polarization gives an isotropic spectrum with a strong Doppler-broadened background. Use of counter-circularly polarized photons in b) eliminates this background as explained in the text.

not observed in benzene [9]. Symmetric anisotropic contributions do appear for all possible photon polarizations. From the results in refs. $[9,10]$ it is clear that the choice of photon polarization allows for the selection of isotropic and anisotropic parts of the spectrum. The Doppler-broadened background can be eliminated if the contribution to the signal strength from the absorption of two photons from one laser beam is small. For $s-s$ transitions in atoms this is easily obtained by using counter circularly polarized (๖૬) light [2]. As it was shown above, the situation is more complicated in molecular spectroscopy, since the anisotropic component of the two-photon tensor leads to an absorption in the circularly polarized ( ২२) light of one laser beam. Fortunately, for $\mathrm{C}_{6} \mathrm{H}_{6}$ and for other aromatic molecules $[8,12]$ this anisotropic component is smaller by nearly one order of magnitude than the corresponding isotropic component of the Q-branch. Since in fig. 3b the Doppler-broadened two-photon absorption from one laser beam can only originate from the anisotropic contribution and therefore is accordingly smaller than the isotropic contribution. As a result the Doppler-broadened background is too small to be observed in fig. $3 \mathrm{~b}$. On the other hand, for pure anisotropic two-photon absorption hands $\left(O, P, R, S-b r a n c h e s\right.$ of an $A_{1 g}$ transition and all branches of $E_{1 \mathrm{~g}}$ or $E_{2 \mathrm{~g}}$ transitions) the best ratio of Doppler-free signal to Doppler-broadened background is obtained for ( ২) polarization conditions [9]. 
Fig. 2 and fig. 3 also differ in the structure of the spectrum even though all the spectra have been taken at the same excitation energy. This is explained by the different Placzec-Teller and Hönl-London rotational factors [10] valid for the isotropic (fig. 3) and anisotropic (fig. 2) part of the spectrum, respectively.

\subsection{Determination of the rotational constants for different vibronic bands}

In this section it will be demonstrated that the spectral resolution and the sensitivity of our set-up is sufficient to quarantee a precise analysis of the rotational structure of different vibronic bands of ben- zene. As it was shown in the preceding section in counter circularly polarized light (2S) the Dopplerfree two-pholon spectrum (see fig. 3b) of a totally symmetric transtion solely consists of the (isotopic) Q-branch. From the selection rules $\Delta J=0$ (Q-branch) and $\Delta K=0\left(\mathrm{~A}_{1 \mathrm{~g}}\right.$ transition symmetry $)$ it follows that the rotational transitions are sorted according to their ground state energy. This makes the assignment of the rotational lines a straight forward procedure if single rotational transitions are resolved. In the analysis of our first Doppler-free measurements [5] we found that it does not suffice to determine one rotational constant for benzene. The general formula for the rotational energy of a rigid symmetric rotor [13] has

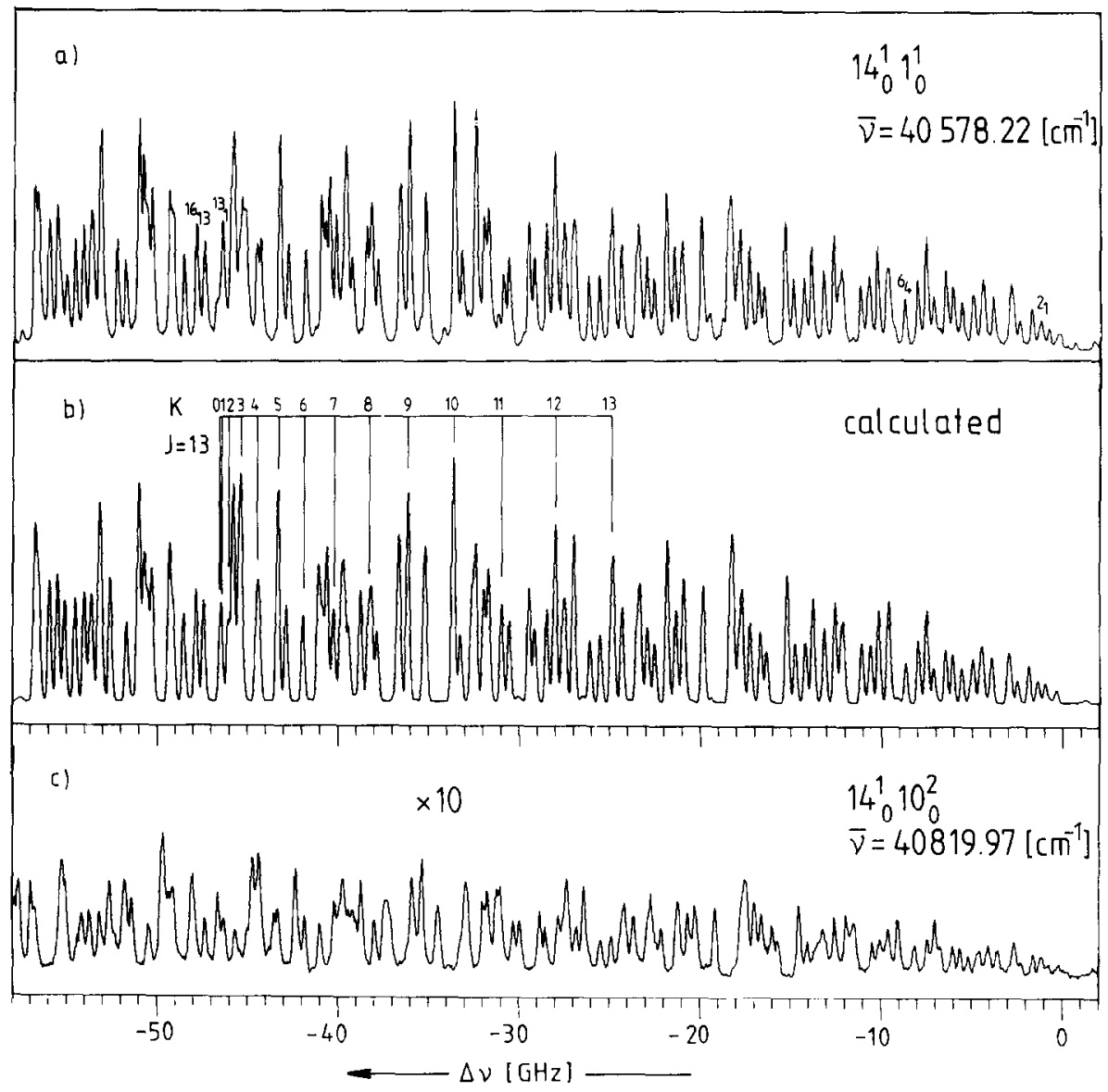

Fig. 4. a) Part of the Q-branch of the $14_{0}^{1} 1_{0}^{1}$ band of $\mathrm{C}_{6} \mathrm{H}_{6}$ (identical with fig. $3 \mathrm{~b}$ ). b) spectrum as calculated with rotational constants from the positions of the lines marked in a). Indicated are the rotational transitions originating from states with $J=13$ and differing $K$ values. c) Corresponding part of the $14_{0}^{1} 10_{0}^{2}$ vibronic band as measured with out set up. Even though this band is weaker by a factor of 20 , the signal to noise ratio is comparable to that in a). 
to be used and in addition to the in-plane constant $B$ the out-of-plane constant $C$ can be found independently from the Doppler-free spectrum. As a result we showed that $C_{\mathrm{v}}^{\prime} \neq \frac{1}{2} B_{\mathrm{v}}^{\prime}$ and that there exists an inertial defect in the excited vibronic state.

Starting from a spectrum calculated with the rotational constants known from Doppler-limited spectroscopy [14] the part of the spectrum with low $J$ is analyzed. In this part of the spectrum the deviation from the experimental result is not too large and the rotational transitions can be identified. First, from the distance of two identified resolved lines with a common $K=1$ but a different $J$, a value for $B_{\mathrm{v}}^{\prime}$ is obtained. Then, from the distance of two neighbouring lines with differing $K$ a value for $C_{\mathrm{v}}^{\prime}$ is calculated. With these values for $B_{\mathrm{v}}^{\prime}$ and $C_{\mathrm{v}}^{\prime}$ again the complete spectrum is calculated and compared with the measured spectrum.

In fig. $4 \mathrm{a}$ the spectrum of the $14_{0}^{1} 11_{0}^{1}$ vibronic band of $\mathrm{C}_{6} \mathrm{H}_{6}$ (identical with fig. $3 \mathrm{~b}$ ) is shown; The frequency of the rotationless transition $(\Delta \nu=0 \mathrm{GHz})$ is $40578.22 \mathrm{~cm}^{-1}$. The measured spectrum covers 2 $\mathrm{cm}^{-1}$ from the $30 \mathrm{~cm}^{-1}$ broad Q-branch. The spectral resolution is found e.g. from the width (FWHM) of the $6_{4}(J=6, K=4)$ line to be $200 \mathrm{MHz}$. This linewidth represents the spectral resolution of the exciting laser light. The resolution is sufficient to resolve most of the rotational transitions of the vibronic band and the refore the assignment procedure is possible as described above. $\Delta B$ is determined from the distance $(45.05 \mathrm{GHz})$ of the $13_{1}$ and $2_{1}$ transitions and then $\Delta C$ is found from the distance (1.4 $\mathrm{GHz}$ ) of the $16_{13}$ and $13_{1}$ transitions which corre- spond to well resolved rotational lines in the spectrum as can be seen in fig. $4 \mathrm{a}$. From $\Delta B$ a value for $B_{\mathrm{v}}^{\prime}$ can be calculated using the most accurate ground state value $B_{0}^{\prime \prime}$ reported [15]. For the determination of $C_{\mathrm{v}}^{\prime}$ the assumption of Pliva and Pine [15], that $C_{0}^{\prime \prime}=$ $\frac{1}{2} B_{0}^{\prime \prime}$ (from the planarity condition) is retained. The results are summarized in table 1 . In fig. $4 \mathrm{~b}$ the corresponding spectrum is shown as calculated from the experimentally found rotational constants. The calculated rotational lines are convoluted with a gaussian linewidth of $210 \mathrm{MHz}$, the observed spectral resolution. Measured and calculated spectra agree well up to roughly $-3 \mathrm{~cm}^{-1}$. First discrepan cies of single lines appear in a nonsystematic manner at $-52 \mathrm{GHz}$. These are probably due to perturbations of the spectrum and a better agreement cannot be reached through the use of another set of rotational constants. In table 1 also the inertial defect of the $14_{0}^{1} 1_{0}^{1}$ band is given as found from the values $B_{\mathrm{v}}^{\prime}$ and $C_{\mathrm{v}}^{\prime}$. It is found to be somewhat smaller than the inertial defect recently found by us for the fundamental vibronic band $14_{0}^{1}$ [5].

In order to demonstrate the sensitivity of the high resolution pulsed light source in fig. $4 \mathrm{c}$ part of the $14_{0}^{1} 10_{0}^{2}$ combination band is shown which has been assigned in previous work [8]. Even though this band is weaker than the $14{ }_{0}^{1} 1{ }_{0}^{1}$ band in fig. $4 \mathrm{a}$ by a factor of 20 the signal to noise ratio in the measured spectrum (fig. 4c) is comparable to that in fig. 4a. Again the blue edge of the isotropic Q-branch under circular polarization $(2 \zeta)$ conditions is shown. Within the first $30 \mathrm{GHz}$ below the rotationless transition the rotational line structure of both totally symmetric vibronic bands

\section{Table 1}

Rotational constants $B_{\mathrm{V}}^{\prime}$ and $C_{\mathrm{V}}^{\prime}$ for various vibronic bands of the first electronically excited state of benzene $\left(\mathrm{C}_{6} \mathrm{H}_{6}\right)$. For the calculation ground state values of Pliva and Pine [15] are used. Also given is the difference $B_{\mathrm{V}}^{\prime} / 2-C_{\mathrm{V}}^{\prime}$ and the inertial defect $\Delta=$ $\hbar / 4 \pi c \cdot(1 / C-1 / B-1 / \beta)$. Owing to the method of determination (compare also ref. [5]) the estimated error of $B_{\mathrm{V}}^{\prime} / 2-C_{\mathrm{V}}^{\prime}$ is much smalle than would be calculated from the errors of $B_{\mathrm{V}}^{\prime}$ and $C_{\mathrm{V}}^{\prime}$

\begin{tabular}{ccccc} 
& $B_{\mathrm{V}}^{\prime}\left(\mathrm{cm}^{-1}\right)$ & $C_{\mathrm{V}}^{\prime}\left(\mathrm{cm}^{-1}\right)$ & $B_{\mathrm{V}}^{\prime} / 2-C_{\mathrm{V}}^{\prime}\left(\mathrm{cm}^{-1}\right)$ & $\Delta\left(\mathrm{amu} A^{2}\right)$ \\
\hline $\left.14_{0}^{1} \mathrm{a}\right)$ & $0.18133(4.5)$ & $0.09074(8)$ & $-7.5 \times 10^{-5}$ & -0.154 \\
$\left.14_{0}^{1} 1_{0}^{1} \mathrm{~b}\right)$ & $0.19122(5)$ & $0.09064(8)$ & $-3.0 \times 10^{-5}$ & -0.062 \\
$\left.14_{0}^{1} 10_{0}^{2} \mathrm{~b}\right)$ & $0.18144(5)$ & $0.09075(8)$ & $-3.0 \times 10^{-5}$ & -0.062 \\
\hline
\end{tabular}
a) Our previous work [5].
b) This work. 
in fig. $4 \mathrm{a}\left(14_{0}^{1} 1{ }_{0}^{1}\right)$ and fig. $4 \mathrm{c}\left(14_{0}^{1} 10_{0}^{2}\right)$ is identical but the spectrum of the $14_{0}^{1} 10_{0}^{2}$ band is somewhat compressed. The values for the rotational constants $B_{\mathrm{v}}^{\prime}$ and $C_{\mathrm{v}}^{\prime}$ are determined as described above and are summarized in table 1.

\section{Summary and conclusion}

In this work we demonstrate that Doppler-free two-photon spectroscopy of polyatomic molecules is possible with intense pulsed laser light of $100 \mathrm{MHz}$ FWHM. This light has been produced by pulsed amplification of cw light from a single mode dye laser.

Since two-photon absorption in this experiment is obtained from one laser source by the absorption of two photons with equal frequencies the problem of a Doppler-broadened background in the spectrum arises. As previously for the case of atoms [2] it is shown in this work for the case of symmetric top molecules that photon polarization strongly controls the Doppler-broadened two-photon absorption. Best experimental conditions for suppressing the Dopplerbroadened background are found to be counter circularly polarized (2S) light in an isotropic totally two-photon transition and in the same sense circularly polarized (22) light in anisotropic two-photon transitions. Using the pulsed light source Dopplerfree two-photon spectra of several vibronic bands in the $\mathrm{S}_{1} \leftarrow \mathrm{S}_{0}$ system of $\mathrm{C}_{6} \mathrm{H}_{6}$ have been obtained. Due to the high peak intensity we were able to measure very weak combination bands with this technique. Hence, it seems also possible to obtain Dopplerfree two-photon spectra of weakly fluorescing molecules or of molecular transitions with low oscillator strength, such as $n \pi^{*}$ transitions.

The $100 \mathrm{MHz}$ spectral resolution of the pulsed laser system is shown to be sufficient for resolving most of the rotational lines in the vibronic spectrum of a molecule as large as benzene. This allows one to investigate in a quantitative way how vibrations influence the rotational constants of a large molecule by rotation-vibration coupling. In addition, perturbations by Coriolis coupling and by Fermi resonances may be studied for molecular states well defined not only in vibrational quanta but also in rotational quanta.

Finally, it is important that a pulsed excitation enables one to measure population lifetimes of the excited molecular states simply by observation of the fluorescence decay. Since, after the Doppler-free highly resolved excitation not only single vibronic states (SVL) but also well defined single rotational states (SVRL) are excited it seems to be possible to investigate in a precise manner how nonradiative processes depend on the rotation of a large polyatomic molecule.

\section{Acknowlegement}

The authors are indebted to Prof. Dr. E.W. Schlag for his permanent interest in this work. They thank the Deutsche Forschungsgemeinschaft for providing the ring-laser system. Valuable experimental help by G. Emslander is gratefully acknowledged.

\section{References}

[1] L.S. Vasilenko, V.P. Chebotayev and A.V. Shishaev, JETP Letters 12 (1970) 113.

[2] F. Biraben, B. Cagnac and G. Grynberg, Phys. Rev. Letters 32 (1974) 643;

M.D. Levenson and N. Bloembergen, Phys. Rev. Letters 32 (1974) 645 .

[3] T.W. Hänsch, K. Harven, G. Meisel and A.L. Schawlow, Optics Comm. 11 (1974) 50.

[4] J.P. Woerdman, Chem. Phys. Letters 43 (1976) 279.

[5] S.V. Filseth, R. Wallenstein and H. Zacharias, Optics Comm. 23 (1977) 231;

A. Timmermann and R. Wallenstein, Optics Comm. 39 (1981) 239.

[5] E. Riedle, H.J. Neusser and E.W. Schlag, J. Chem. Phys, 75 (1981) 4231.

[6] R. Wallenstein and T.W. Hänsch, Optics Comm. 14 (1975) 353.

[7] M.M. Salour, Optics Comm. 22 (1977) 202.

[8] L. Wunsch, H.J. Neusser and E.W. Schlag, Chem. Phys. Letters 31 (1975) 433;

L. Wunsch, F. Metz, H.J. Neusser and E.W. Schlag, J. Chem. Phys. 66 (1977) 386.

[9] W. Hampf, H.J. Neusser and E.W. Schlag, Chem. Phys. Letters 46 (1977) 406.

[10] F. Metz, W.E. Howard, L. Wunsch, H.J. Neusser and E.W. Schlag, Proc. R. Soc. Lond. A363 (1978) 381.

[11] P.R. Monson and W.M. McClain, J. Chem. Phys. 53 (1970) 29.

[12] U. Boesl, H.J. Neusser and E.W. Schlag, Chem. Phys. 15 (1976) 167.

[13] G. Herzberg, Molecular spectra and molecular structure, Vol. III (Van Nostrand, Princeton, 1966).

[14] J.R. Lombardi, R. Wallenstein, T.W. Hänsch and D.M. Friedrich, J. Chem. Phys. 65 (1976) 2357.

[15] J. Pliva and A.S. Pine, J. Mol. Spectrosc. 93 (1982) 209. 